\title{
Re: Noninvasive Methods to Evaluate Bladder Obstruction in Men
}

\author{
Dean S. Elterman, Bilal Chughtai, Richard Lee, Alexis E. Te, Steven A. Kaplan \\ James Buchanan Brady Department of Urology, Weill Cornell Medical College of Cornell University, New \\ York, USA
}

\section{To the editor,}

Recently, we received the last volume of IBJU which first article is a review entitled "Noninvasive methods to evaluate bladder obstruction in men" by D.S. Elterman et al.

Reading this paper leads to some thoughts.

Our main concerns are first that from paragraph "Symptoms" to paragraph "Doppler Resistive Index", this paper looks like the paper of M. Belal and P. Abrams "Noninvasive methods of diagnosing bladder outlet obstruction in men. Part 1: Nonurodynamic approach" J Urol 2006; 176: 22-8 (not referenced by the authors) and second, more important, that the authors have made a mixture between two methods the condom method (paragraph Measurement using External Catheter (MEC)) and the penile cuff method (paragraph Measurement Using Penile Compression (MUCP)). Some results about penile cuff method are included in the paragraph external catheter and vice versa.

About external catheter, the authors mention a good accuracy for weak flow rate while the original paper mention a lack of accuracy if the flow rate is lower than $5.4 \mathrm{~mL} / \mathrm{s}$ (van Mastright et al. Development and application of the condom catheter for non-invasive measurement of bladder pressure. J Urol 2009; 25: 99-104).

The description of penile compression given by the authors is (only) that of the cuff deflation (Mc Rae et al NAU 1995; 14: 101-114 and Gleason et al. NAU 1997; 16: 93-100) which is not used today. The penile cuff method currently used is an inflation-deflation method (C.J. Griffiths et al.); after initiation of voiding, a cuff is progressively inflated at a stepwise increment of $10 \mathrm{~cm}_{2} \mathrm{O}$ to a maximum of $200 \mathrm{~cm} \mathrm{H}_{2} \mathrm{O}$ until interruption of the flow. Thus many measurements are possible. The surge of flow observed when the cuff is deflated allows calculating a PCR index (see next remark).

In addition, some non-invasive methods have been forgotten: the Penile Compression and Release (PCR) from M.P. Sullivan et al. (NAU 1995; 14: 481-3 \&t NAU 2000; 19: 657-669) allowing calculating a PCR index, and the D index of voiding dysfunction from F.A. Valentini et al. (BJU Int 2008; 101: 995-9) obtained from a modelled analysis of free uroflows.

An additional concern is the finding of some references errors.

Reference 40 ascribed to MEC is a study of the penile cuff deflation method. Reference 41 ascribed to MEC is a study of the penile cuff inflation-deflation method. These two references are not related to the use of a condom. 
Reference 43 ascribed to MUCP is a study of the condom method and is not related to the Penile Compression method.

The second appearance of Reference 40 (in paragraph MUCP) is an error as differences of height between pressure transducer and the cuff are not investigated in that paper. I think that the authors mistake the study of Mc Rae et al. for the well known paper of C.J. Griffiths et al. ("A nomogram to classify men with lower urinary tract symptoms using flow and noninvasive measurement of bladder pressure". J Urol 2005; 174: 1323-6).

Due to all these mistakes and inaccuracies, we think that that article must be revised.

Correspondence address:
Françoise A. VALENTINI, MD, PhD and
Pierre P. Nelson, MD, PhD
Service de Médecine Physique et Rééducation (Neurologie)
Hôpital Rothschild,
5, rue Santerre, 75012 - Paris, France.
Fax: + 331 4019-3656
E-mail: favalentini@gmail.com

\section{REPLY BY THE AUTHORS}

We thank the reader for the thoughtful comments and the critical review of our manuscript. In our attempt to consolidate the techniques and review various methods to evaluate bladder outlet obstruction there may have been confusion and in the description of the techniques of the condom method and penile cuff method. As the reader mentions, there is lack of accuracy in lower flow rate below $5.4 \mathrm{~mL} /$ sec as described by van Mastright et al. Also, as the reader pointed out, we failed to mention the penile compression and release described from Sullivan et al. It also seems there was an error in our reference manager that led to our failure to cite M. Belal and P. Abrams. Their review was excellent in describing several techniques and was fundamental to our understanding of several noninvasive methods of measuring bladder outlet obstruction. We appreciate the feedback on our review and sincerely apologize for our oversight on these inaccuracies in these methods. We look forward in the future to expand our investigations of these technologies in a broader cohort of men. Also look at potentially confounding variables such as obesity and neurogenic bladder dysfunction. Thank you again.

\section{Respectfully,}

The authors 